\title{
AGENT BASED ENERGY EFFICIENCY IN WSN
}

\author{
Amar Jukuntla ${ }^{1}$, Venkatesulu D ${ }^{2}$ \\ ${ }^{1}$ Department of CSE, VFSTR, India \\ ${ }^{2}$ Department of CSE, VFSTR, India \\ ${ }^{1}$ ja_cse@vignan.ac.in, ${ }^{2} \mathrm{drdv} \_$cse@ vignan.ac.in
}

\begin{abstract}
A large number of Wireless Sensor Network (WSN) devices are used to monitor the data from the environment and many applications such as disaster management, monitoring, military, healthcare and security etc. are used to monitor the environment. In these networks sensor nodes transmission rate is high and it leads to limit the battery power. This causes network redundancy and network life time will not be prolonged. In this paper, this can be addressed by using with the help of agents. Here, agents are used to collect data from the cluster head instead of forwarding directly to the mobile sink. When data collection is done, agents will transmit data to the mobile sink. This may reduce energy consumption and prolongs the network life in WSNs. Along with agents, existing techniques are also presented in this paper with comparative study.
\end{abstract}

Keywords - Agents, Mobile Sink, power constraints, life time, energy efficiency

\section{INTRODUCTION}

WSN is one of the fastest growing area in networking. It consist of huge number of sensor nodes which are deployed in specific applications. These applications are having their own specific functionalities such as military applications, home intelligence, industrial process control, healthcare applications, environment monitoring, etc. All these sensor nodes(SN) have small in size but does communication, computation and sensing capabilities. These SN collaborate among themselves to accomplish the common task with short range radio signals[1,2]. But, SN have limited capacity in terms of processing resources, bandwidth, power, memory and limited life time. The goal of SN is to sense environmental parameters like temperature, heat, light and forwards the data to the sink in query form from targeted phenomena. Energy is consumed less compared to the data transmission in WSN[3]. Sensed data is aggregated using aggregated function and forward the data to the sink, due to this more energy can be saved instead of sending individuals nodes. DA is a process of gathering and consolidating the data which are useful for effective communication. Because this effective communication among nodes will depend on the aggregate techniques used. Data aggregation is fundamental process for saving the limited energy resources. Data aggregation can enhance network lifetime and energy efficiency. 


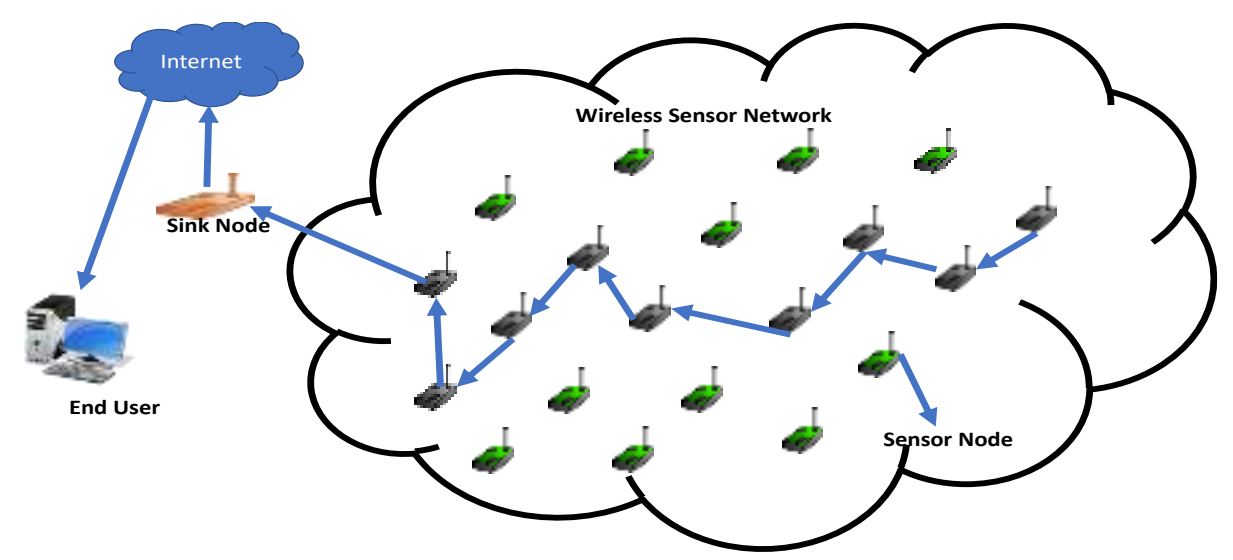

Fig. 1 WSN

DA is used to aggregate sensory data which are collected from various $\mathrm{SN}$ and data aggregation algorithms are used to aggregate sensed data coming from the different SN. These aggregate algorithms will take sensor readings from various $\mathrm{SN}$ as an input and generates aggregated data as an output. Routing protocols are used for selection of best path for forwarding data to the sink to source node. In WSNs, failure rate is more due to dynamic environment, where WSNs are deployed, but it is not sufficient to send the data with single path. In order to solve this problem multi path is used to discover the destination and if one path fails to send data to the destination, can select another path, which is discovered. So, routing protocol is designed to solve this problem for efficient communication in terms of power consumption, performs the data aggregation between the source and destination by discovering multiple paths between them. Suitable data aggregation technique is selected based on the application that it involves.

\section{DATA AGGREGATION}

Processing of data with the help of aggregation approaches is called data aggregation(DA). These algorithms uses the data which are collected from sensor node and does data processing (aggregates) using aggregation algorithms like LEACH, Tree based DAT, Adaptive DAT, Cluster based DAT, etc,. Aggregated data is forwarded to the mobile sink through efficient path. Many aggregation reviews are presented here, some approaches are explained in this section.

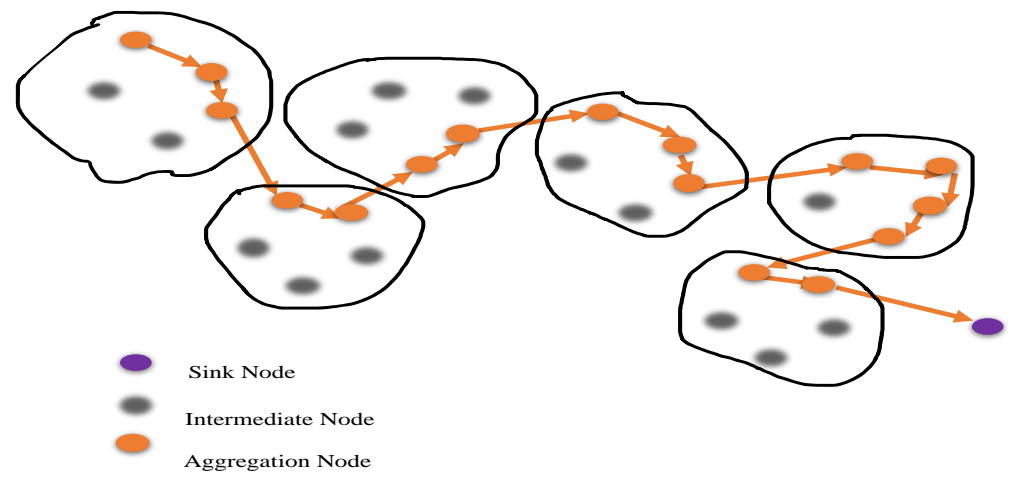

Fig. 2 Aggregation Process 


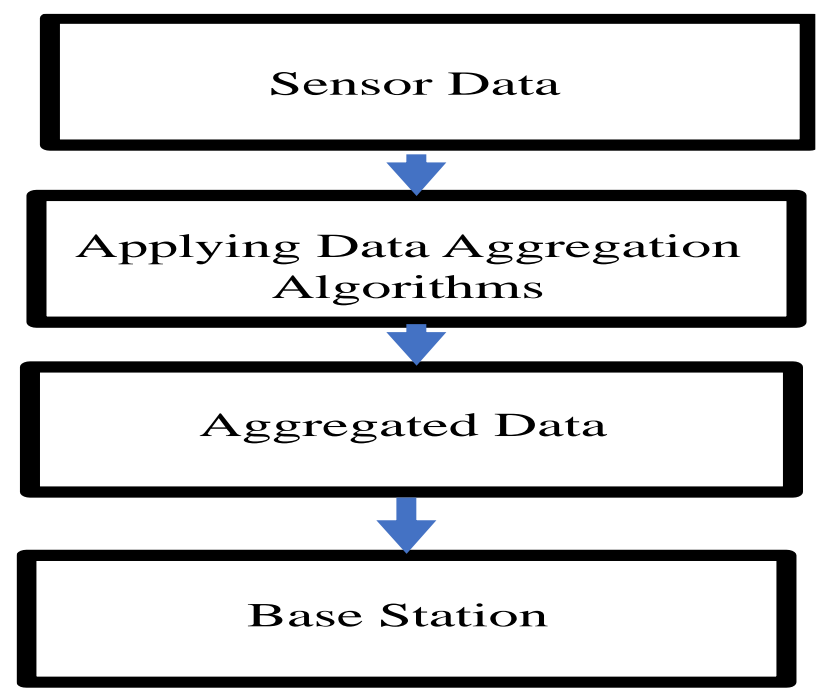

Fig. 3 Data Aggregation Flow Chart

\subsection{CENTRALIZED DATA AGGREGATION TECHNIQUES}

This is a centralized data communication approach, where all nodes sends data to central node through shortest path using multihop routing protocols. The SN forwards the data packet to leader node and it aggregates the data and queried. Enormous number of messages are forwarded for a query and it is equal to the sum of external paths for each node in best case. These nodes sends data to leader node via child nodes which are present in the network.

\subsection{DATA AGGREGATION USING CLUSTER}

In WSNs, clusters were formed based on the division of network and each cluster has one leader(cluster head), which is elected by members in the cluster. Cluster head computes data aggregation and result is transmitted to the sink. This process saves life time of the network.

\subsection{DA USING TREE BASED NETWORK}

In tree based network, DA done at intermediate node when sensor nodes are arranged in tree like a spanning tree, and sink node act as root and leaf nodes are considered as source node. Parent node transmits the data flow starts from leaf nodes up to the sink and data aggregation done at parent nodes.

\section{COMPARISON OF DA APPROACHES}

Comparison of DA approaches are very difficult, due to different kinds of DA techniques are used, there lack of bench marks. Comparison will be done for finding effective technique in data aggregation.

Table I. Comparison of DA Techniques

\begin{tabular}{|l|l|l|l|}
\hline \multicolumn{1}{|c|}{$\begin{array}{c}\text { Data Aggregation } \\
\text { Technique }\end{array}$} & Routing Protocol & \multicolumn{1}{|c|}{ Merit } & Demerit \\
\hline $\begin{array}{l}\text { Adaptive Data } \\
\text { Aggregation Energy and } \\
\text { latency }\end{array}$ & Multihop & $\begin{array}{l}\text { Power } \\
\text { consumption } \\
\text { reduced }\end{array}$ & $\begin{array}{l}\text { Life time is less } \\
\text { in network }\end{array}$ \\
\hline
\end{tabular}




\begin{tabular}{|c|c|c|c|}
\hline Adaptive DA- Cluster & Hierarchical & $\begin{array}{l}\text { Head overload is } \\
\text { reduced on overall }\end{array}$ & $\begin{array}{ll}\text { Lesser } & \text { data } \\
\text { accuracy } & \end{array}$ \\
\hline $\begin{array}{l}\text { Cluster Based DA-Leader } \\
\text { Selection Scheme }\end{array}$ & Chain & $\begin{array}{l}\text { Improves the } \\
\text { energy efficiency } \\
\text { and network life } \\
\text { time }\end{array}$ & $\begin{array}{l}\text { Communication } \\
\text { cost are not } \\
\text { considered }\end{array}$ \\
\hline NL based DA-Energy & Chain & $\begin{array}{l}\text { It reduces the } \\
\text { energy cost }\end{array}$ & $\begin{array}{l}\text { Larger } \\
\text { latency }\end{array}$ \\
\hline Tree based DA-Hybrid & Tree & $\begin{array}{l}\text { Improved in } \\
\text { accuracy }\end{array}$ & $\begin{array}{l}\text { Lesser network } \\
\text { lifetime }\end{array}$ \\
\hline
\end{tabular}

\section{CLOUD BASED WSN FOR DA}

In WSNs, design of applications are very difficult, because these will depend on network routing protocols and network devices. Data can be gathered very carefully and managed efficiently. So that, it can be used in future different purposes. Managed and stored data can be accessed easily in future when it is required but there is not enough storage for gathered data for long time decisions which are used in future. Additional resources are required to manage data to process large amount of data in order to save time and cost.

To overcome this problem, cloud data centers can be established which can improve the reliability, data efficiency etc. This cloud centers are not only used for data aggregation, which analyses and store the data coming from different sensor nodes used in WSNs. This approach uses clustering mechanism for gathering the data and applies classification techniques to classify the data in different ways to process and to use it and processed data is efficiently to perform data aggregation and to store data in cloud servers for future use. For classification, can use different types of classification algorithms are used for processing the data.

This cloud based DA, will fail when gathered data is more, it can't hold and system may go down. For this highly reliable resources can be utilized and maintained by some soft computing techniques like fuzzy.

\section{SOFT COMPUTING TECHNIQUES FOR WSN}

In WSN, soft computing techniques are also used for DA, like swarm-based data aggregation, genetic, neural-networks and fuzzy based data aggregation.

\subsection{GENETIC ALGORITHM BASED DA TECHNIQUE}

In view of the transformative thoughts, Genetic Algorithm(GA) is an adaptive heuristic search algorithm and it has rapid growth in AI. For navigation purpose, huge amount of search spaces are allocated. This is used for finding optimal solutions.

On mirroring the nature, activities such as selection, cross over, mutation and accepting the issues are using in the GA's. Different types of GA's are used for prolonging the WSN's life time.

i. NH GA-ABC based energy efficient clustering[5], which provides energy consumption and which does not give secure routing information and accurate values.

ii. In Data aggregation and routing in WSN[6], which removes the redundancy and reduces the energy efficiency and increases the life time of a node. It will not give accurate measures and values for the security purpose are also not considered. 


\subsection{NEURAL-BASED DA TECHNIQUE}

The utilization of a lot of sources of info should create the ideal set of yields in neural system (NN). The quality of associations is set utilizing different techniques. First strategy, is used to set the loads expressly based on prior knowledge. Second strategy is used for teaching patterns to the $\mathrm{NN}$ and permitting it to change loads as indicated by some learning guideline. For learning situations in NN classification is practiced, which include different types of learnings like: reinforcement, unsupervised and supervised learning. In these techniques, when more nodes are kept in inactive (sleep) state, energy savings can be done significantly.

i. Data aggregation and authentication protocol[7], accuracy is highly obtained by NN and life time of sensor nodes are increased with the help of backbone propagation in $\mathrm{NN}$, but this backbone propagation induces the overload. Hence this approach is not considered for routing in WSNs.

ii. GHHECR protocol[8], it will not give accurate values for routing, because of insecure routing for data aggregation.

\subsection{FUZZY-BASED DA TECHNIQUE}

A fuzzy based data aggregation techniques are used energy efficiency, some of the few papers short description are given below.

i. Data fusion algorithms(DFA) [9], it consumes less computational power and energy is saved effectively. For extraction of final accurate results, few data samples are required and it will not reduce cost value due to absence of energy consumption.

ii. Event-oriented data aggregation (EDA)[10], energy consumption is less, when data is delivered and satisfies the application target when event is activated. It doesn't give accurate measured values and does not consider the security measures while doing the data aggregation.

\subsection{SWARM-BASED DATA AGGREGATION TECHNIQUE}

Swarm-based technique or ACO imitates the behavior of ants. Ants finds the shortest path from the sustenance source to the home (nest), with the assistance of the pheromone which is released by ants. The shortest path is guided by the pheromone which fills in as correspondence media among the ants. Target path is related to the trails of rich pheromone.

Nature-inspired data gathering protocol[11], this protocol is fully distributed, scalability, reliable and substantial energy savings. This mechanism is used to predict values accurately but security measures are not available.

\section{PROPOSED APPROACH}

This section illustrates multi agent model for data aggregation in wireless sensor networks. Proposed approach consists of 1. Formation of sensor network, 2. Data collection using Agents.

\subsection{FORMATION OF SENSOR NETWORK}

Wireless Sensor Network has deployed $\mathrm{n}$ sensors in a specified environment and all sensor nodes are identified with their own ID's and they have fixed location or can't move once network is established. All sensor nodes are distributed uniformly and randomly over the network. It maintains transceiver which maintains maximum transmission range and adopts the multihop transmission for communication. An undirected graph $\mathrm{G}=(\mathrm{V}, \mathrm{E})$ is used for calculating the range of the network. Sensory data 
is collected through the sensor nodes with the help of the SN's are known as intermediate senor nodes.

Wireless Sensor Network is divided into clusters and data is forwarded to mobile sink when it approaches to the cluster [12]. When intermediate nodes receives the beacon messages, all nodes are started transmitting data to the cluster head. But in this approach data transmission is not done directly, agents were sent for collecting the data from each cluster and transmits to sink node. Data collection using agents will be explained in following section.

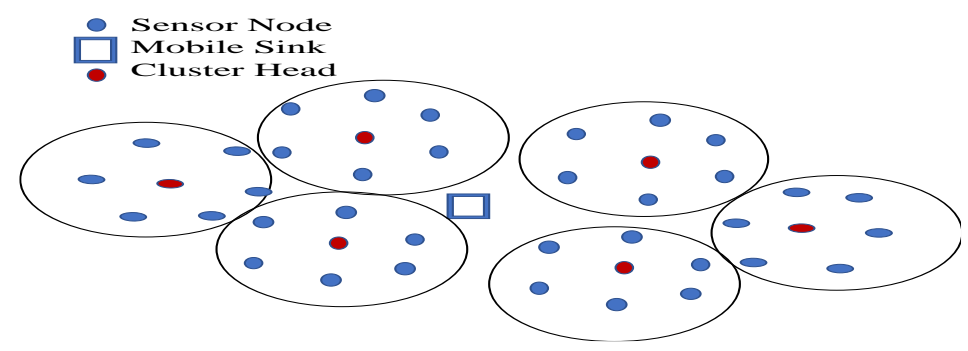

Fig. 4 WSN with cluster heads

\subsection{DATA COLLECTION USING AGENTS}

Wireless Sensor Network, completes its organization of clusters formation and routing between the nodes. Whenever sink node approaches to the clusters, it dispatches the agents to collect the data from the cluster heads. These cluster heads started the data collecting from the intermediate nodes in the cluster. These intermediate nodes start transmitting the data to the cluster head at a time. In this process, energy decreases due to huge transmission at a time, to the cluster head and some sensor nodes may go out of energy.

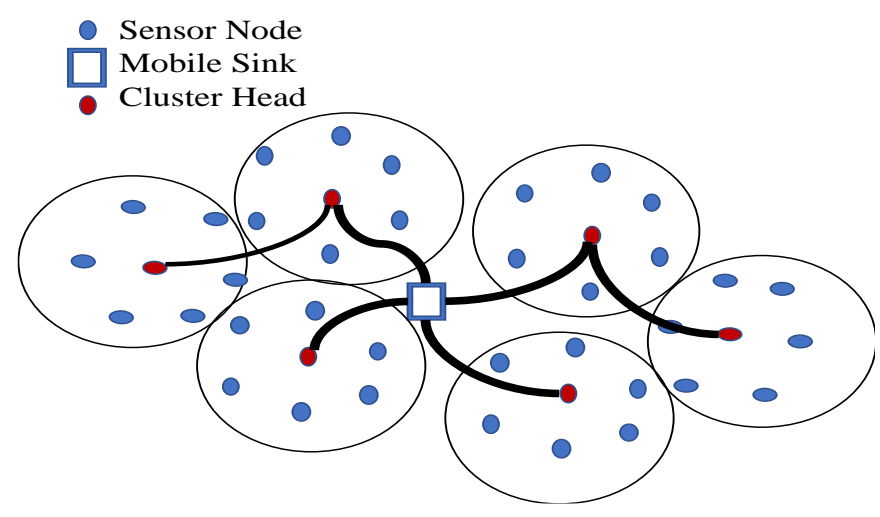

Fig. 5 WSN with Path

For handling this situation, ACO wakeup scheduling [12] is used and collecting data from intermediate nodes those are in active mode. Some sensor nodes will go in inactive mode, due to out of energy and neighbor nodes are in active mode which transmits the data to cluster head whenever it required and it goes to sleep mode and activates the sleeping nodes when it runs out of energy.

Computing network life time, entire WSN monitoring area is divided into $\mathrm{n}$ cluster is

$$
\beta=\frac{A}{n}
$$


Where $\bar{A}$ is number of active sensor nodes in a cluster, $\bar{n}_{\text {number of clusters in a }}$ monitoring area, $\beta=1$ there should be 1 active sensor node should cover the monitoring area. Otherwise, network is not covered.

This entire process is done before agent visiting the cluster head. Whenever agent visits the cluster head first time in the cluster, it started receiving the data from the active nodes. If agent reaches the last cluster head in the network, it starts collecting the data from the cluster head in its way back to the sink. This way consumes the less energy and spends less time for collecting data in the network.

In this approach agent is sent to each cluster only, when path is defined in the network from one cluster to another cluster.

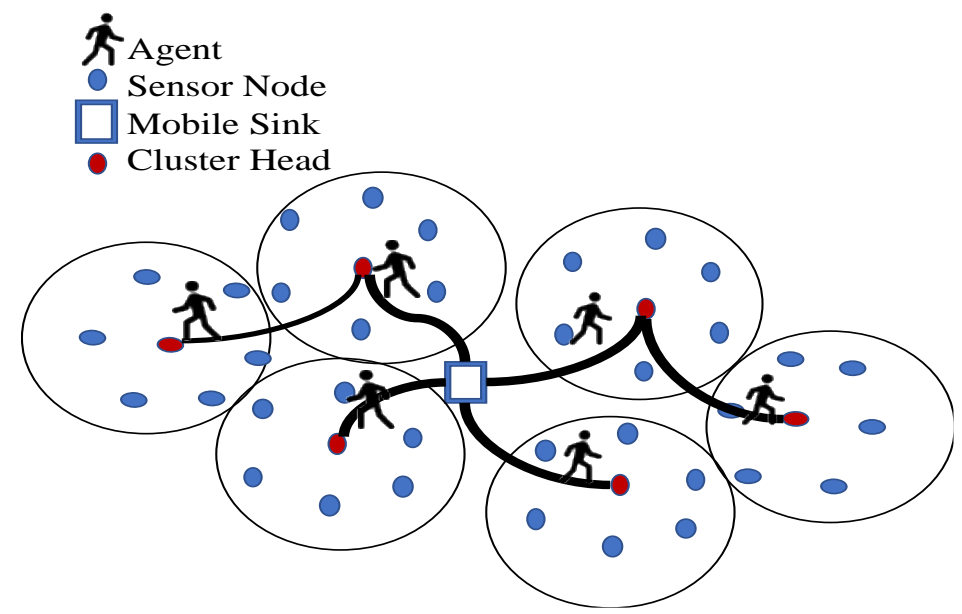

Fig. 6 Mobile Sink dispatches Agents

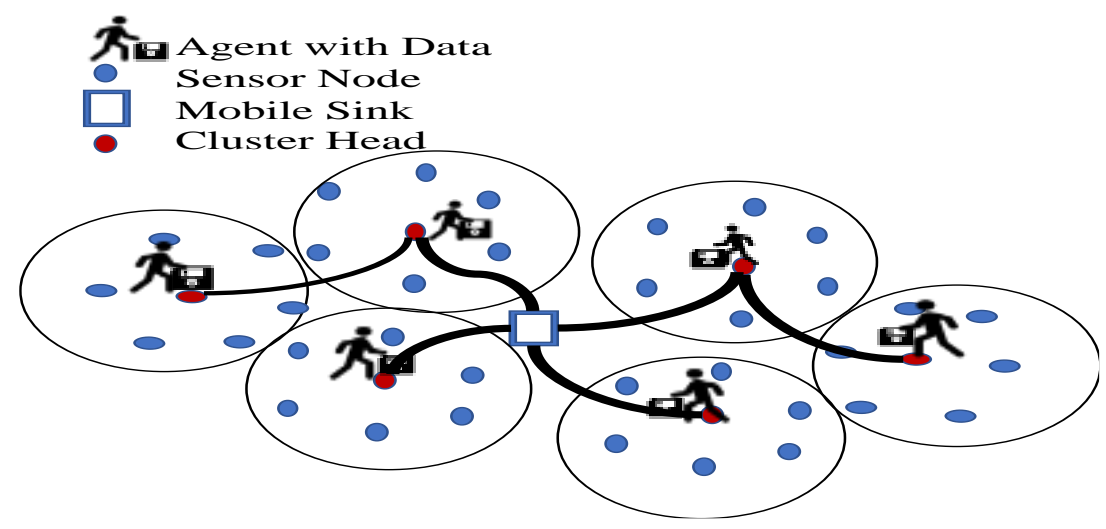

Fig. 7 Agent collecting data from $\mathrm{CH}$ 


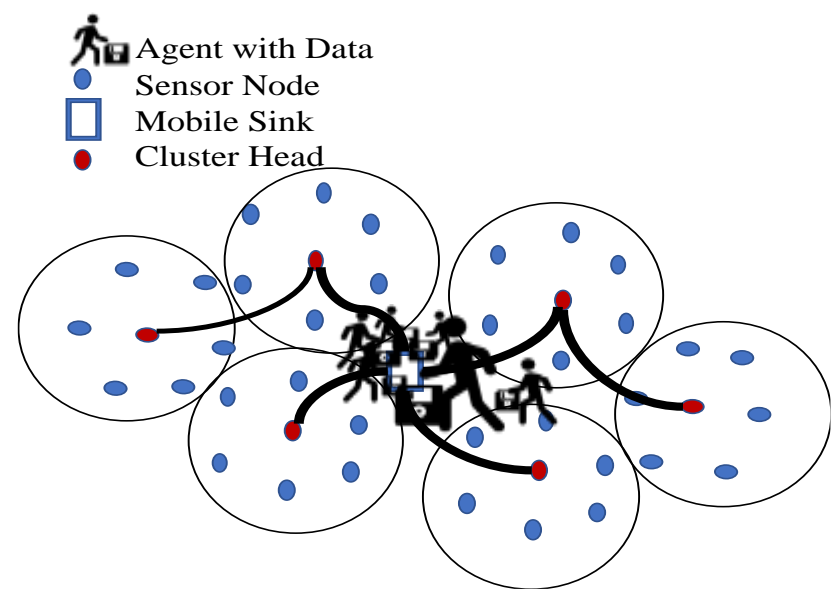

Fig. 8 Mobile Sink collecting data from Agents

\section{ALGORITHM}

Step 1: Form the clusters

Step 2: Elect cluster head in the cluster

Step 3: Use wakeup scheduling protocol among the sensor nodes.

Step 4: Find the routing information with in the cluster, in that all sensor nodes should transmit data to the cluster head.

Step 5: Agent visits the each cluster head until it reaches the end of the network, until rout is defined.

Step 6: When Agent reaches last cluster in the network, it starts collecting the data from the cluster head in its return back journey to the mobile sink.

Step 7: Agent's data collection is done, it deliver data to the mobile sink.

Step 8: Exit

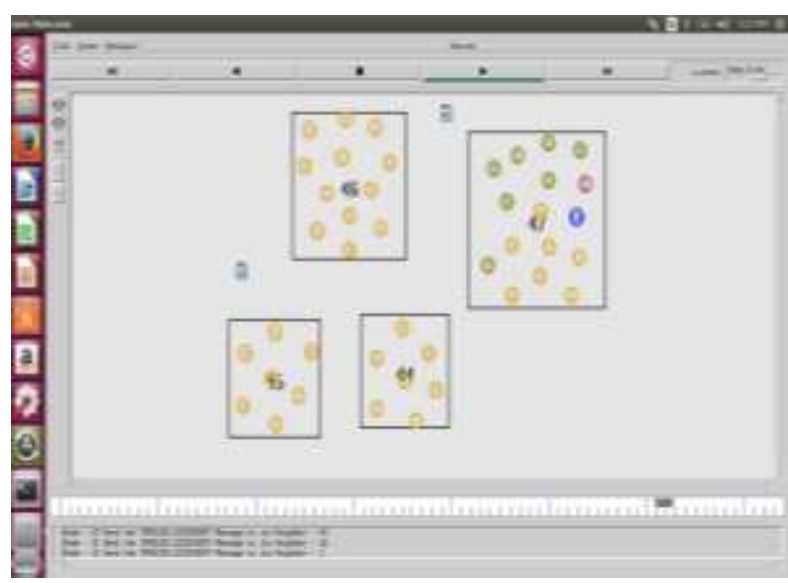

Fig. 9 Cluster formation in WSN 


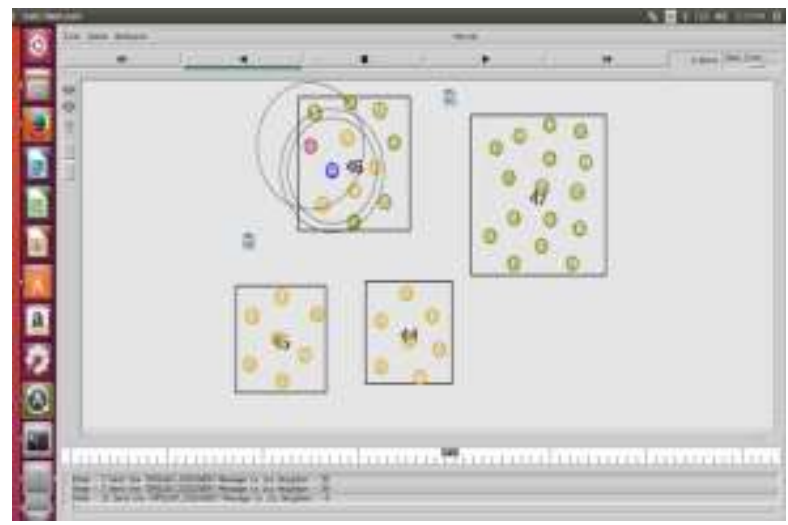

Fig. 10 Cluster Head selection within a Cluster

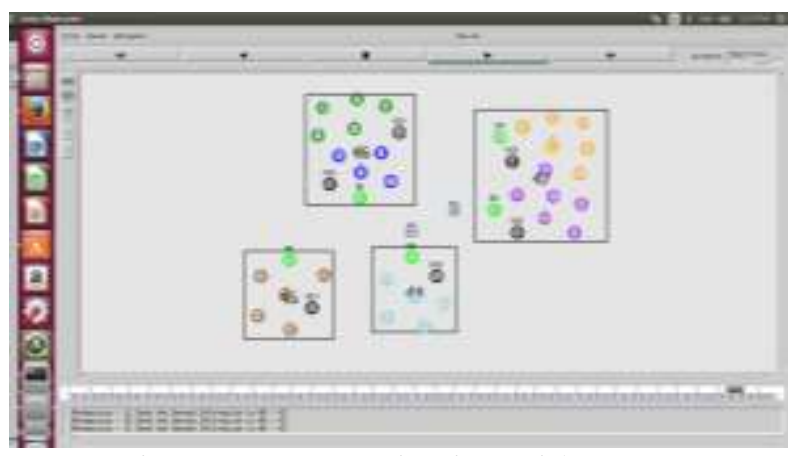

Fig. 11 Communication with Agent

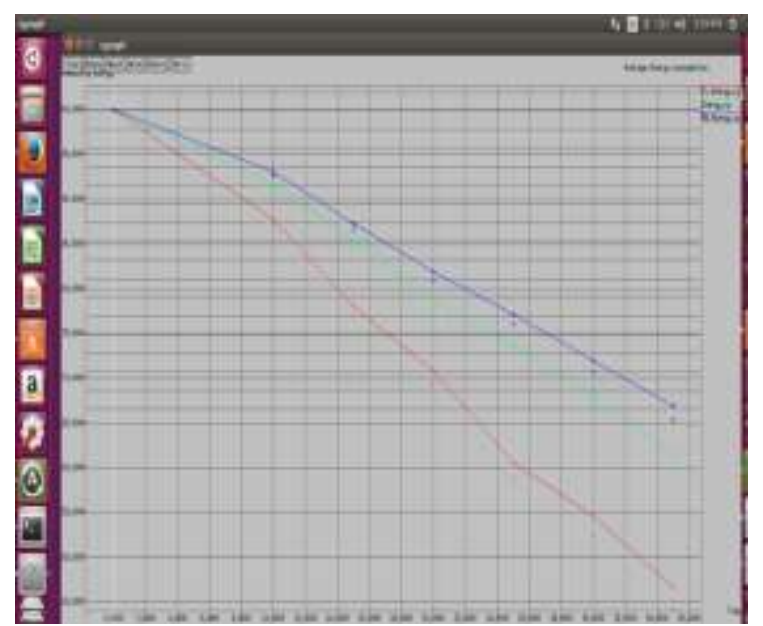

Fig. 12 Energy consumption graph with Agents

\section{CONCLUSION}

In this paper, we addressed problems in wireless sensor networks and presented a novel solution to this with the help of agent. These are used to collect data from the cluster head which are present in the cluster. Agents will visit cluster heads only when path is fully defined and then only agents are dispatched from the mobile sink to gather the data from the cluster. This may prolong network life time and compared to other existing approaches, which are presented in this paper. In future will use some AI based techniques to address the WSN problems.

\section{REFERENCES}

[1] Tan, H. O, Korpeoglu, I. "Power efficient data gathering and aggregation in wireless sensor networks." ACM SIGMOD Record, 32. 4(2003): 66-71. 
[2] Pourpeighambar, S. B., Aminian, M., \& Sabaei, M. "Energy efficient data aggregation of moving object in wireless sensor networks" In Australasian telecommunication networks and applications conference (2011): 1-8.

[3] Krishnamachari, L., Estrin, D., \& Wicker, S. "The impact of data aggregation in wireless sensor networks." In Proceedings of 22 nd international conference of distributed computing system work (2002): 575-578..

[4] Chen, H, Mineno, H., \& Mizuno, T. "Adaptive data aggregation scheme in clustered wireless sensor networks." Computer Communications, 31.15(2008): 3579-3585.

[5] Mehrjoo, S, Aghaee, H, Karimi, H. "Novel hybrid GA-ABC based energy efficient clustering in wireless sensor network." Can. J. Multimedia Wirel. Netw. 2.2(2011): 41-45 32.

[6] Al-Karaki, J, Ul-Mustafa, R, Kamal, E.A. "Data aggregation and routing in wireless sensor networks: optimal and heuristic algorithms”, Int. J. Comput. Telecommun. Netw. 53.7(2009): 945-960.

[7] Jakhar, N, Nandal, R. "A secure data aggregation approach in WSN Using ANN", Int. J. Res. Eng. Appl. Sci., 2.2, (2012): 2249-3905.

[8] Jaleel, U, Chaturvedi, A. "GHHECR protocol for energy conserving routing in sensor networks." Int. J. Comput. Appl. 13.3, (2011): 1-4.

[9] Su, W, Bougiouklis, T. "Data fusion algorithms in cluster-based wireless sensor networks using fuzzy logic theory." Proc. 11th WSEAS Int. Conf. on Communications, Agios Nikolaos, Crete Island, Greece, (2007): 26-28.

[10] Guo, Y, Hong, F, Guo, Z., Jin, Z, Feng, Y. "EDA: event-oriented data aggregation in sensor networks." IEEE 28th Int. Conf. on Performance Computing and Communications Conf., (2010): 25-32.

[11] Selvakennedy, S, Sinnappan, S, Shang, Y. "T-ANT: a nature-inspired data gathering protocol for wireless sensor networks", J. Commun., 1.2 (2006): 22-29.

[12] Amar Jukuntla, Keerthi p, "SAWR: Scheduling Algorithm for Wireless Sensor Networks with Rendezvous Nodes", 2015 IEEEICCIC, Madhurai.

[13] Aman Kumar and Rahul Hans, "Performance Analysis of DSDV, I-DSDV, OLSR, ZRP Proactive Routing Protocol in Mobile Ad Hoc Networks in IPv6", International Journal of Advanced Science and Technology, SERSC Australia, ISSN: 2005-4238 (Print); 2207-6360 (Online), vol. 77, April (2015), pp. 25-36, http://dx.doi.org/10.14257/ijast.2015.77.03.

[14] Ahmed Salim, Asmaa Ahmed Badran, "Impact of using Mobile Sink on Hierarchical Routing Protocols for Wireless Sensor Networks", International Journal of Advanced Science and Technology, SERSC Australia, ISSN: 2005-4238 (Print); 2207-6360 (Online), vol. 77, April (2015), pp. 37-48, http://dx.doi.org/10.14257/ijast.2015.77.04.

[15] Jianning Han, Shuai Tang, Yu Liu, Peng Yang and Lulu Li, "Research on Band Gap Algorithm and Regularity of Two Component Phononic Crystals", International Journal of Advanced Science and Technology, SERSC Australia, ISSN: 2005-4238 (Print); 2207-6360 (Online), vol. 110, January (2018), pp. 33-44, http://dx.doi.org/10.14257/ijast.2018.110.04.

[16] Jina Hong and Namgi Kim, "An Efficient Power Management for Wireless Sensor Systems", International Journal of Control and Automation, SERSC Australia, ISSN: 2005-4297 (Print); 22076387 (Online), vol. 8, no. 1, January (2015), pp. 275-286, http://dx.doi.org/10.14257/ijca.2015.8.1.25.

[17] Kamlendu Kumar Pandey and Dr. S.V. Patel, "Development of an Effective Routing Protocol for Cluster Based Wireless Sensor Network for Soil Moisture Deficit Monitoring", International Journal of Control and Automation, SERSC Australia, ISSN: 2005-4297 (Print); 2207-6387 (Online), vol. 8, no. 1, January (2015), pp. 243-250, http://dx.doi.org/10.14257/ijca.2015.8.1.22.

[18] Chayoung Kim and Jinho Ahn, "Causal Order Protocol Based on Virtual Synchronous Group Membership in Wireless Sensor Networks", International Journal of Control and Automation, SERSC Australia, ISSN: 2005-4297 (Print); 2207-6387 (Online), vol. 8, no. 2, February (2015), pp. 9-20, http://dx.doi.org/10.14257/ijca.2015.8.2.02.

[19] Yuchul Jung, Jinyoung Kim, Heung-Seon Oh, Dongjun Suh, Jeong-Soo Kim, Seok-Hyong Lee, KwangYoung Kim and Jungsun Yoon, "Entity-Driven Knowledge Analytics Platform for Science and Technology", International Journal of Advanced Science and Technology, SERSC Australia, ISSN: 2005-4238 (Print); 2207-6360 (Online), vol. 116, July (2018), pp. 1-12, http://dx.doi.org/10.14257/ijast.2018.116.01.

[20] Mohit Balu, Gulshan Kumar and Se-Jung Lim, "A Review on Security Techniques in Vanets", International Journal of Control and Automation, NADIA, ISSN: 2005-4297 (Print); 2207-6387 (Online), vol. 12, no. 4, April (2019), (pp. 1-14), http://dx.doi.org/10.33832/ijca.2019.12.4.01.

[21] N. Thirupathi Rao, Debnath Bhattacharyya, V. Madhusudhan Rao and Tai-Hoon Kim, "Black Hole Attacks on WSNs Using Discrete Simulator: An Extensive Review", International Journal of Grid and Distributed Computing, NADIA, ISSN: 2005-4262 (Print); 2207-6379 (Online), vol.11, no.12, December (2018), (pp. 33-42), http://dx.doi.org/10.14257/ijgdc.2018.11.12.03.

[22] Amine El Hadi, Rachid El Ayachi, Mohammed Erritali and Mohamed Baslam, "Improved Results of an OCR via NLP using MapReduce Framework", International Journal of Grid and Distributed Computing, NADIA, ISSN: 2005-4262 (Print); 2207-6379 (Online), vol.11, no.11, November (2018), (pp. 13-28), http://dx.doi.org/10.14257/ijgdc.2018.11.11.02. 